Research Article

\title{
Knowledge-Based Structure Optimization Design for Boom of Excavator
}

\author{
Shuan-Qiang Yang $\mathbb{D}^{1,2}$ Xin-Long Huang, ${ }^{1}$ Zhen-Hui Shen, ${ }^{1}$ and Yang-Mei Zhang ${ }^{1}$ \\ ${ }^{1}$ Fujian Jiangxia University, Fuzhou 350108, China \\ ${ }^{2}$ Dalhousie University, Halifax, Canada \\ Correspondence should be addressed to Shuan-Qiang Yang; yangshq@fjjxu.edu.cn
}

Received 16 September 2020; Revised 15 March 2021; Accepted 8 August 2021; Published 21 August 2021

Academic Editor: Vasilios Spitas

Copyright ( $) 2021$ Shuan-Qiang Yang et al. This is an open access article distributed under the Creative Commons Attribution License, which permits unrestricted use, distribution, and reproduction in any medium, provided the original work is properly cited.

\begin{abstract}
During the design optimization of the excavator boom, there are many design variables and complicated processes. The original optimization methods mainly focused on the optimization of mathematical models, and they lacked consideration in the use of domain knowledge, design-specification knowledge, expert experience knowledge, and historical examples. In order to comprehensively utilize the domain knowledge and expert experience knowledge, this study uses the optimization process analysis, uses knowledge expression and coding processing technology to encode the boom structure, builds an optimal design coding system based on knowledge guidance, and realizes the automatic optimization design of the boom structure. In the process of constructing the knowledge-oriented optimization system, to realize the reuse of the knowledge of the boom structure design in the numerical optimization iteration, a knowledge processing flowchart of the boom structure design is constructed. The concept of "shape distance" is proposed to judge the similarity feature matrix of the boom structure coding. To evaluate whether the stress distribution is uniform, a fast prediction model based on stress characteristic regions is constructed. The research results show that, under the comprehensive consideration of the four working conditions, the knowledge-guided optimization of the boom structure can avoid the deformity in the optimization process, accelerate the calculation speed of the optimization model, and improve the optimization quality of the model.
\end{abstract}

\section{Introduction}

Excavator is a commonly used mechanical device in engineering; it is widely used in hydraulic engineering, transportation, power engineering, mining, and other mechanical construction; it can easily excavate several tons of Earth; therefore, the design of this part is the key to the overall performance of the excavator. The structural design and optimization process should be fully integrated application of domain knowledge, expert experience knowledge. In order to achieve a more reasonable design of the excavating structure, many scholars have adopted various methods for optimization. Wu et al. [1] proposed a single-objective nonlinear optimization model, which was optimized by combining genetic algorithm with MATLAB, and adopted a multiobjective nonlinear model to improve the convergence speed and calculation accuracy. Zhang et al. [2] used programming language and finite element analysis software to model the research object and used annealing genetic algorithm combined with secondary development technology to optimize the structural size of the model. Compared with the traditional genetic algorithm, this method has better optimization effect and higher efficiency. Aderiani et al. [3] used the topological structure to express the structural form and iterated the expression through the genetic algorithm to optimize the overall structure and size. Migvei [4] proposed HS (coordinated search) and FA (firefly algorithm) for complex structure optimization problems and confirmed that these two search algorithms can complete global optimization in a short time, Wendt et al. [5] proposed a knowledge-based genetic algorithm to guide the structural genetic optimization process using design domain knowledge. 
In recent years, methods such as approximate models $[6,7]$, structure reduction order modeling $[8,9]$, collaborative optimization $[10,11]$, and constraint expression and processing [12], all make use of improvements in numerical algorithms or combine static system knowledge with numerical optimization algorithms. These methods have improved the optimization rate and the optimization results to a certain extent, but they have not been able to extract and use the group target knowledge and constraint knowledge generated in the optimization process to guide the structural optimization. It is impossible to minimize the problem of global optimization time for each generation of optimized group structure, and it is easy to fall into a locally optimal solution. For this reason, it is necessary to make full use of domain knowledge, design specifications, expert experience, and other knowledge to study the structural intelligent numerical optimization methods guided by the knowledge of the optimization process. By extracting and using the knowledge of the optimization process to guide the structural numerical optimization process, a comprehensive optimization model is established. Taking the boom of excavation as an example, this paper proposes a structural optimization design method that combines a numerical optimization algorithm with a knowledge system. The integrated technology of numerical calculation and logical reasoning is discussed, and a method of guiding numerical optimization by logical reasoning is studied.

\section{State of the Art}

Many scholars have done a lot of research work on the optimal design of the boom. Xu et al. [13] proposed an improved multiobjective evolutionary algorithm (MOEA) based on decomposition by establishing a multiobjective optimization model with three kinematic indicators and two mechanical indicators as objective functions; the core components of the excavator are optimized. Qiu et al. [14] proposed a new modeling strategy based on the combination of hinge point size variable and working device hinge point force, studied with a variety of alternative models, and optimized the structure design of excavator with a bionic intelligent optimization algorithm, achieving excellent results. Li et al. [15] proposed the optimal design of a hydraulic excavator working device, based on the parallel particle swarm optimization (PPSO), through the establishment of the kinematics and dynamics analysis model of hydraulic excavator. The improved parallel PPSO algorithm is used to develop the optimal design for the excavator working device. Zhu et al. [16] proposed a method to comprehensively integrate Pro/E and ANSYS with ISIGHT optimization platform to carry out multiobjective collaborative optimization of the boom. The hybrid optimization strategy of DOE and gradient optimization is adopted to explore design problems intelligently, and new initial values are selected continuously for automatic simulation and optimization. Zou et al. [17] proposed an optimized design method for the full parameter working attachment of a hydraulic excavator to reduce weight and minimize stress. The method employs a novel 3D force output model to fully and accurately calculate the maximum force and moment that the excavator can apply in the preferred bucket force space. Based on this model, the joint force acting on the working attachment is calculated, a fully parametric geometric model of the working attachment is constructed, and the hybrid variable genetic algorithm is used to solve the optimization problem, which enables mechanical devices to be optimized during the design process.

In the above research results, the core components of the excavator are mainly optimized by different optimization models. There is no in-depth combination of related knowledge such as knowledge areas, design specifications, and expert experience. Deviations in the optimization results cannot be avoided during the optimization process. This paper uses a genetic algorithm (GA) and a knowledge-based system (KBS) model algorithm. Based on the consideration of multiple operating conditions, heuristic search speeds up the optimization convergence speed and achieves global optimization. It uses KBS logical reasoning ability. It can effectively identify malformed structural solutions generated in each generation of GA population and optimize the malformed structural solutions, thereby improving optimization efficiency and solution quality.

The remainder of this paper is organized as follows. Section 3 is a detailed analysis and description of the knowledge-guided optimization design method. Section 4 gives an illustrative example to verify the feasibility and effectiveness of the excavating boom structure optimization recognition technology and the excavating boom structure parameter adjustment technology. Conclusions are summarized in Section 5.

\section{Methodology}

3.1. Knowledge-Based Optimization Design Method. The boom is a large part of the excavator, which is a large consumption of materials and energy consumption. The greater the volume of material consumed, the greater the mass. So, the boom is designed as small as possible under the condition of satisfying the engineering application.

In order to minimize the volume of the boom, the stress distribution should be more uniform according to the idea of equal strength design. To evaluate the stress distribution, the boom of the excavator structure is divided into several stress characteristic regions according to the stress distribution characteristics. The smaller the local maximum stress difference of each specific stress region is, the more uniform the stress distribution is. Therefore, the optimization design of the boom structure is to integrate the four calculation conditions specified in GB9141-88 and to minimize the volume of the boom structure and the most uniform stress distribution without increasing the maximum stress value of the structure; the material selected in this article is Q345, and the objective function can be expressed as 


$$
\begin{aligned}
\min f(X) & =\frac{V(X)}{V}+\sum_{i=1}^{N} \frac{f_{i}(X)}{F} \\
f_{i}(X) & =\sigma_{m}-\sigma_{i}, \quad i=1,2, \ldots, N \\
\sigma_{m} & =\max \left\{\sigma_{1}(X), \sigma_{2}(X), \ldots, \sigma_{i}(X)\right\}, \quad i=1,2, \ldots, N
\end{aligned}
$$

where $X=\left(x_{1}, x_{2}, \ldots, x_{n}\right) \in R^{n}$ is the optimal design variable for the $n$-dimensional boom structure, $V$ is the initial material volume, $V(x)$ is the boom material volume, $F$ is the sum of the stress difference in the initial state, $f_{i}(x)$ is the difference between the maximum stress $\sigma_{m}$ of the structure in comprehensively calculating the four working conditions and the local maximum stress $\sigma_{i}$ of the $i$ th stress characteristic area, and $i$ is the number of specific regions.

The constraint system optimized for the boom structure mainly includes structural strength constraints, geometric shape constraints, motion interference constraints, and stability constraints. The main reason for the existing methods to generate abnormal structure during the solution process is that the geometric shape constraints are not adequately expressed. Therefore, a dynamic design optimization method for integrating the genetic algorithm (GA) with the knowledge-based system (KBS) is proposed. GA implements efficient numerical calculations, speeds up optimization convergence, and achieves global optimization through heuristic search. KBS realizes the acquisition, expression, and processing of domain knowledge, designspecification knowledge, expert experience knowledge, and historical instance knowledge. By utilizing the logical reasoning ability of KBS, it can effectively identify the malformed structural shapes generated in each generation of GA groups and optimize the malformed structural shapes to improve the optimization efficiency and the quality of the solution. The knowledge-guided boom structure optimization design workflow is shown in Figure 1.

In the early stage of the operation of genetic algorithms, there may be a very high degree of fitness among a small number of individuals in the population. When the fitness calculation method is used for selection operations, these individuals will be reproduced in large numbers and may eventually flood the entire population. Individuals with low fitness will be eliminated prematurely in the group, and the diversity of the group will be reduced, resulting in premature maturity of the group, and the genetic algorithm will converge to a locally optimal solution in advance. On the contrary, in the later stage of the operation of the genetic algorithm, the population is becoming more and more concentrated, the differences between most individuals and the best individuals are small, and the competitiveness between them is weakened, causing the probability that individuals are selected to the next generation is similar, and the evolution process lost competitiveness and degenerated into a random selection process. Therefore, in order to avoid the premature phenomenon at the initial stage of the iteration and the degradation phenomenon at the later stage of the iteration, in the initial stage of the iteration, the difference between the fitness of individuals with higher fitness and other individuals can be reduced, and in the later stage of the iteration, the difference between the fitness of the best individual and the fitness of other individuals can be enlarged. Improve the competition among individuals and ensure the diversity of the group. Construct the following fitness function $f(x)$ as follows:

$$
\begin{gathered}
F(X)=\frac{[\sqrt[v]{t}]}{f(X) \times C}, \\
v=1+\ln D,
\end{gathered}
$$

where $[\sqrt[v]{t}]$ represents an integer value not greater than $\sqrt[v]{t}, D$ is the maximum evolution algebra set according to the complexity of the problem, $t$ is the current evolution algebra, and $C$ is an adjustment coefficient, which is used to adjust the range of $F(X)$ to avoid $F(X)$ being too large or too small.

In order to obtain the instance base of intelligent optimal design of actuators and make the case inference result match the structure and performance of the design target, the grey correlation degree is used to evaluate the correlation degree of structural performance between different actuators. Let the variable value of the boom structure solution to be designed be $X_{0}=\left[x_{0}(1), x_{0}(2), \ldots, x_{0}(n)\right]$, and the known parameters of the $M$ boom instances in the instance library are

$$
\left\{\begin{array}{l}
X_{1}=\left[x_{1}(1), x_{1}(2), \ldots, x_{1}(n)\right] \\
X_{2}=\left[x_{2}(1), x_{2}(2), \ldots, x_{2}(n)\right] \\
\ldots \ldots \\
X_{M}=\left[x_{M}(1), x_{M}(2), \ldots, x_{M}(n)\right]
\end{array}\right.
$$

Then, the correlation coefficient is $\zeta_{i}(k)$ between $X_{i}(i=$ $1,2, \ldots, M)$ and $X_{0}$ under the $k$ th $(k=1,2, \ldots, M)$ parameter.

$$
\zeta_{i}(k)=\frac{\min _{i} \min _{k}\left|x_{0}(k)-x_{i}(k)\right|+\varepsilon \cdot \max _{i} \max _{k}\left|x_{0}(k)-x_{i}(k)\right|}{\left|x_{0}(k)-x_{i}(k)\right|+\varepsilon \cdot \max _{i} \max _{k}\left|x_{0}(k)-x_{i}(k)\right|},
$$

where $\varepsilon$ is the resolution coefficient, and the value is between 0 and 1 , usually 0.5 . Therefore, the matching degree $r_{0 i}$ between the $i$ th boom instance and the boom to be designed is

$$
r_{0 i}=\frac{1}{n} \cdot \sum_{k=1}^{n} \zeta_{i}(k)
$$

3.2. Knowledge Expression and Processing. The structural parameters of the gooseneck double-acting hydraulic cylinder type boom are shown in Figure 2.

There are many design constraints on the boom structure, and the coupling relationship between variables is powerful. By using the inheritance and similarity between the typical boom structures, the idea of parts' classification in group technology is introduced, and the expression and 


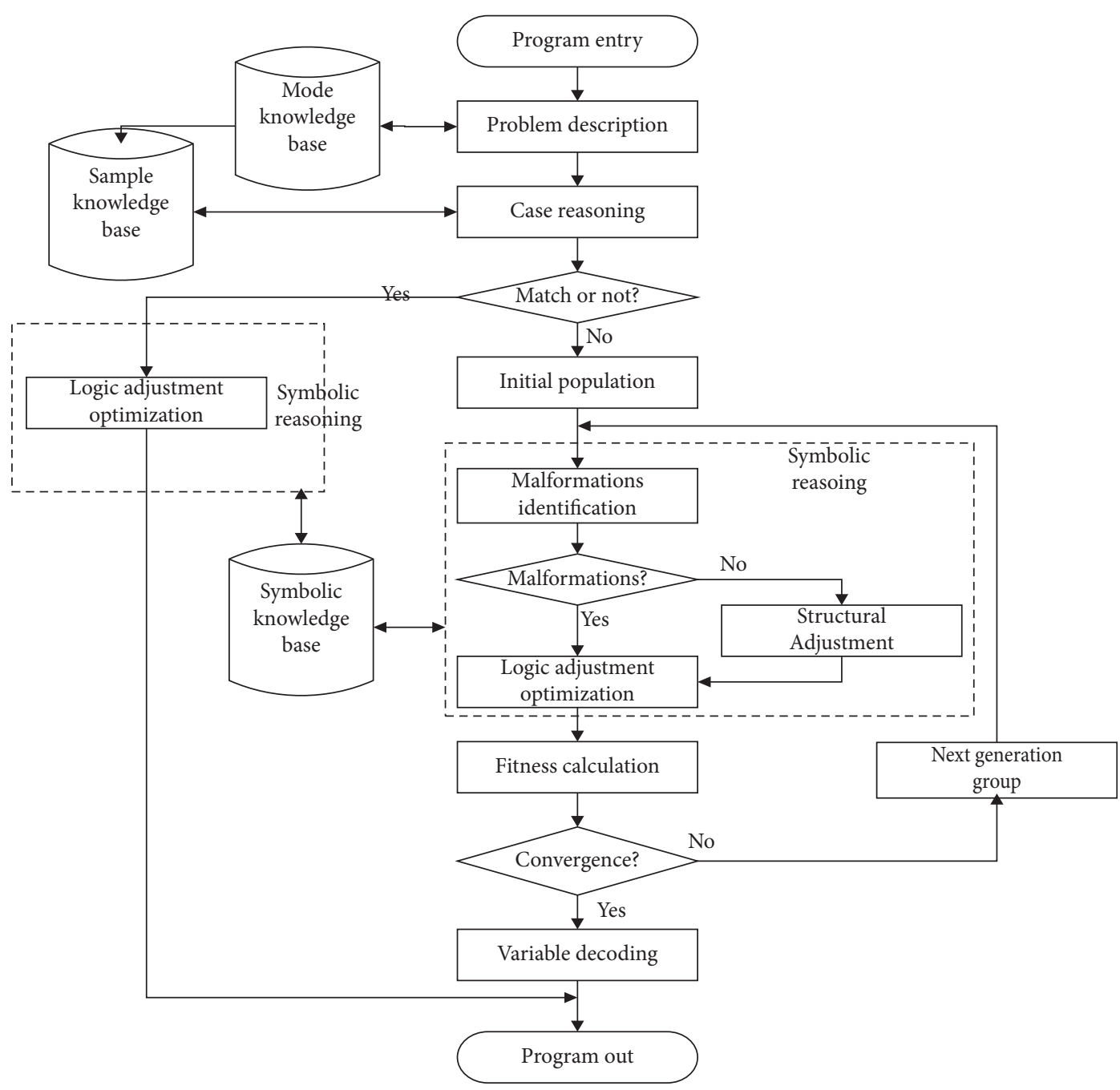

FIgURE 1: Workflow chart of the intelligent optimization design.
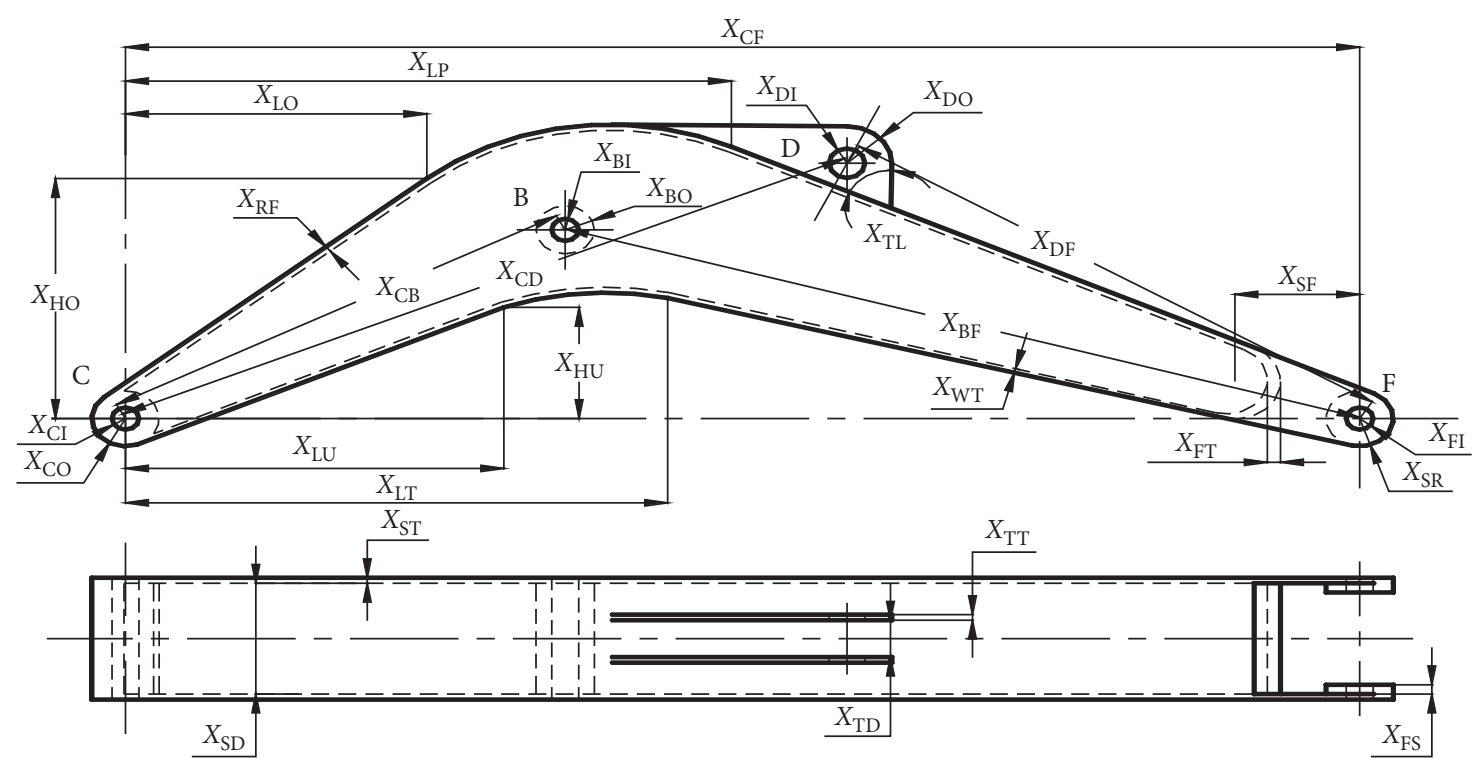

Figure 2: Structure parameters. 
processing of the design of the boom structure is realized by encoding and classifying the boom structure. If the boom structure generated during the optimization process cannot be attributed to any of the regular categories, it indicates that the boom structure is a malformed structure.

As shown in Figure 3, corresponding to the hierarchy of boom structure design process, the boom structure coding includes a name category code, a structure shape code, and a detailed design code. The code bit has a total of 28 bits, and the value is $1-28$. The code value is expressed in hexadecimal, and the value is $0 \sim \mathrm{D}$. The first to second digits are the name category codes, which are used to indicate the standard bucket capacity and the overall structure. The coding shape is shown in Table 1. The third to seventh digits are the structure shape codes, and the coding shape is shown in Table 2. The 8 th to 28 th bits are detailed design codes, and the coding method is shown in Table 3. Among them, the 8th to 13 th bits are shaped design codes, which represent the shape characteristics of the boom structure. The 14th to 28th bits are the size design codes, which indicate the value of the boom structure size.

With the aid of the coding system, not only the boom entity can be coded quickly but also the structure code can be used to reverse the range of values of each structural parameter. A similarity feature matrix expresses the range of values of each code bit of the same type of boom structure coding. The similarity characteristic matrix of the $k$ th type boom is expressed as

$$
S^{(k)}=\left|s_{i j}^{(k)}\right|_{I \times J},
$$

where $J$ is the structure coding length, and each column of the similarity feature matrix has a one-to-one correspondence with each code bit of the structured coding, $J=28, I$ is the maximum value of all code bits in the coding of the boom structure, $I=13$ (hexadecimal D), and there is one structure code under this category which satisfies that the $j$ th code bit value is $i, s_{i j}^{(k)}=1$, otherwise $s_{i j}^{(k)}=0$.

In the boom optimization process, the main reason for the malformation of the boom structure is that the shape design code is unreasonable. Therefore, the correct classification of the shape design code is the core to ensure that the boom structure will be reshaped during the optimization process. According to a large number of design examples, through cluster analysis, the shape design category of a whole gooseneck bending boom with a double-boom hydraulic cylinder is eight categories, and the corresponding similarity characteristic matrix is shown as

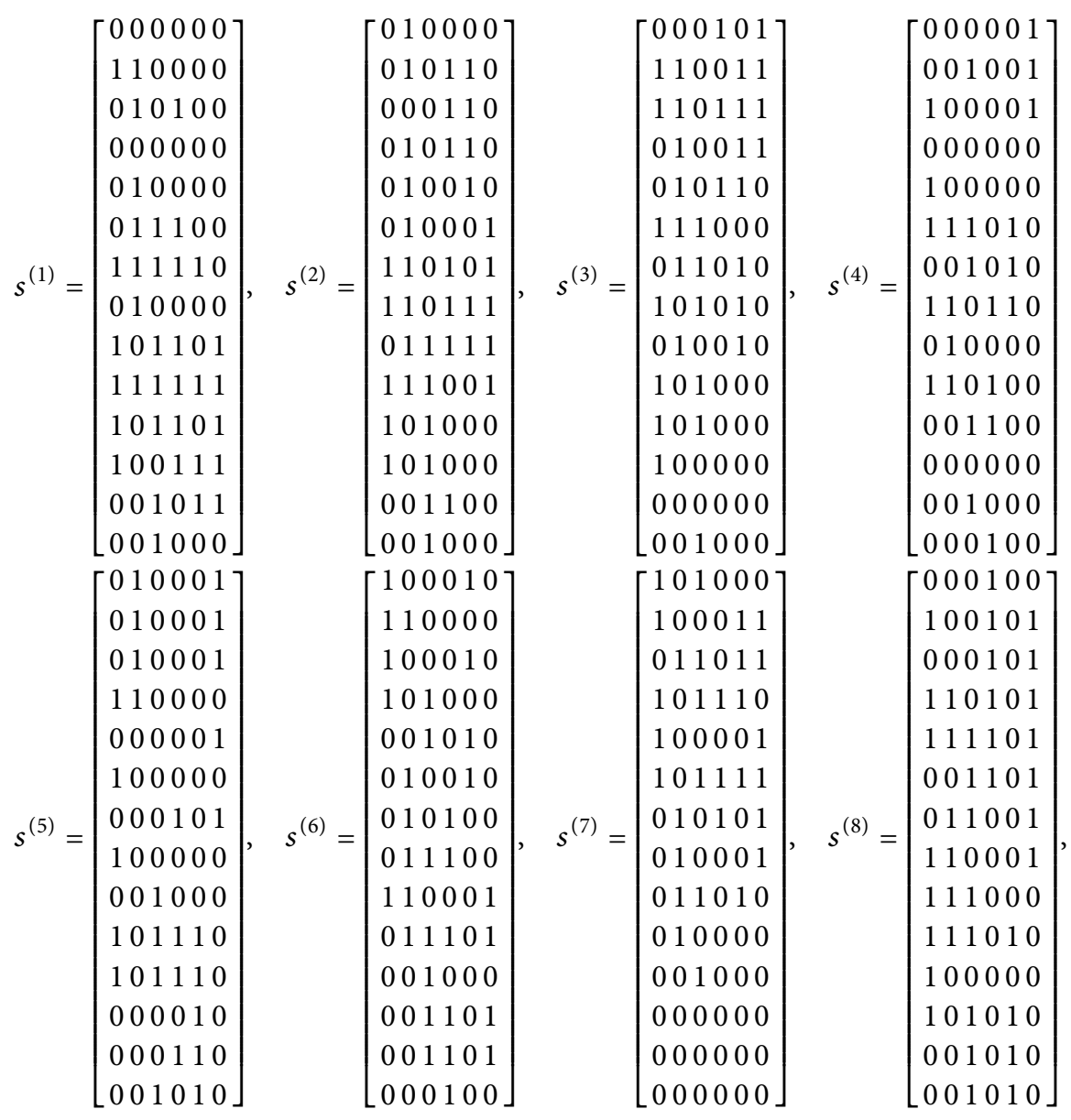




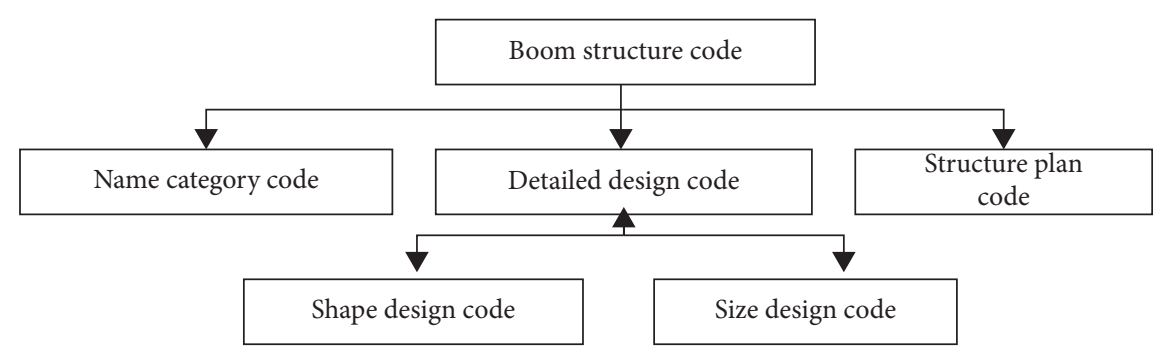

FIGURE 3: Coding composition of the boom structure.

TABLE 1: Name of the category code.

\begin{tabular}{lccccccccccccccc}
\hline \multirow{2}{*}{ Code bits } & \multirow{2}{*}{ Meaning } & \multicolumn{1}{c}{ Code value } \\
& & 0 & 1 & 2 & 3 & 4 & 5 & 6 & 7 & 8 & 9 & $\mathrm{~A}$ & $\mathrm{~B}$ & $\mathrm{C}$ & $\mathrm{D}$ \\
\hline 1 & Standard bucket volume & 0.1 & 0.12 & 0.16 & 0.2 & 0.25 & 0.4 & 0.5 & 0.6 & 0.8 & 1 & 1.25 & 1.6 & 2 & 3 \\
2 & Integral structure & Integral & Combination & - & - & - & - & - & - & - & - & - & - & - & - \\
\hline
\end{tabular}

TABLE 2: Structure shape code encoding.

\begin{tabular}{|c|c|c|c|c|c|c|}
\hline \multirow{2}{*}{$\begin{array}{l}\text { Code } \\
\text { bits }\end{array}$} & \multirow{2}{*}{ Meaning } & \multicolumn{5}{|c|}{ Code value } \\
\hline & & 0 & 1 & 2 & 3 & 4 \\
\hline 3 & $\angle B F D$ & $\leq 15^{\circ}$ & $>15^{\circ}$ & - & - & - \\
\hline 4 & $\angle \mathrm{BCF}-\angle \mathrm{DCF}$ & $\leq-5^{\circ}$ & $>-5^{\circ} \sim 0^{\circ}$ & $>0^{\circ}$ & - & - \\
\hline 5 & $\begin{array}{c}\text { Rough classification of } \\
\text { shapes }\end{array}$ & Straight boom & Bent boom & - & - & - \\
\hline 6 & $\begin{array}{c}\text { Detailed classification of } \\
\text { shapes }\end{array}$ & $\begin{array}{c}\text { No subdivision } \\
\text { shape }\end{array}$ & Gooseneck shape & $\begin{array}{l}\text { Three- } \\
\text { section }\end{array}$ & $\begin{array}{l}\text { Connecting rod } \\
\text { connection }\end{array}$ & $\begin{array}{c}\text { Bolt } \\
\text { connection }\end{array}$ \\
\hline 7 & $\begin{array}{c}\text { Number of hydraulic } \\
\text { cylinders }\end{array}$ & $\begin{array}{l}\text { Single hydraulic } \\
\text { cylinder }\end{array}$ & $\begin{array}{l}\text { Double hydraulic } \\
\text { cylinder }\end{array}$ & - & - & - \\
\hline
\end{tabular}

where the row corresponds to the code value $1 \sim \mathrm{D}$, the column corresponds to the code bit $8-13$, and the row corresponds to the code value $0 \sim \mathrm{D}$. In order to judge whether the structure code $\mathrm{C}$ belongs to the similarity feature matrix $S^{(k)}$, the concept of "shape distance" is proposed, and the calculation method is as follows:

$$
d^{(k)}=\sqrt{\sum_{j=1}^{J}\left[\min \left\{\left.\left|s_{i j}^{(k)}-c_{j}\right|\right|_{i=1,2, \cdots, I}\right\}\right]^{2}}
$$

where $c_{j}$ is the value of the $j$ th bit of C. If $d^{(k)}=0$, the boom belongs to the $k$ th class. If there is $d^{(k)} \neq 0$ for all categories, the boom structure is malformed. The smaller $d^{(k)}$, the closer the structure of the boom to the $k$ th type boom. In order to reuse the knowledge of the design of the boom structure in the optimization iteration, the flowchart of constructing the knowledge of the design of the boom structure is shown in Figure 4.

For the boom entity randomly generated in the optimization process, the "shape distance" is used to identify whether the boom structure is malformed. For the normal boom, do not make any adjustments to the structural parameters, directly output the code of the boom and its category. For the malformed structure boom, in order to maintain the randomness of the value in the optimization, the boom structure shape in the category corresponding to the minimum shape distance is used as a template, and the code position in the malformed structure that is inconsistent with the template code is shifted to the regular value interval, which is randomly adjusted to normalize its shape.

3.3. Variable Selection and Knowledge Acquisition. In order to evaluate whether the stress distribution of the boom structure is uniform, a rapid prediction model of the stress distribution based on the stress characteristic region is constructed.

As shown in Figure 5, the boom has six stress characteristic regions [18], and the corresponding local maximum stress is $\sigma=\left[\sigma_{\mathrm{I}}, \sigma_{\mathrm{II}}, \sigma_{\mathrm{III}}, \sigma_{\mathrm{IV}}, \sigma_{\mathrm{V}}, \sigma_{\mathrm{VI}}\right]$. The boom structure is complex and has many dimensional parameters. In order to reduce the dimension of the optimized design variables and improve the solution speed, the structural parameters with large Spearman rank correlation coefficient of $\mathrm{V}(X)$ and $\sigma_{\mathrm{m}}$ are selected as the optimal design variables [19], and the optimal design variables are determined as $X=\left[X_{\mathrm{ST}}, X_{\mathrm{HU}}\right.$, $\left.X_{\mathrm{SD}}, X_{\mathrm{RF}}, X_{\mathrm{WT}}, X_{\mathrm{LT}}, X_{\mathrm{LO}}, X_{\mathrm{BO}}, X_{\mathrm{CO}}, X_{\mathrm{SR}}, X_{\mathrm{TT}}, X_{\mathrm{HO}}, X_{\mathrm{DO}}\right]$.

The Spearman rank correlation coefficient between $X$ and $S$ is shown in Table 4. Combining the correlation between $X$ and $V(X)$, the following conclusions can be drawn:

(1) If $X_{\mathrm{HU}}$ or $X_{\mathrm{LO}}$ is increased, then $\sigma_{\mathrm{m}}$ will be increased and $V(X)$ will be decreased 


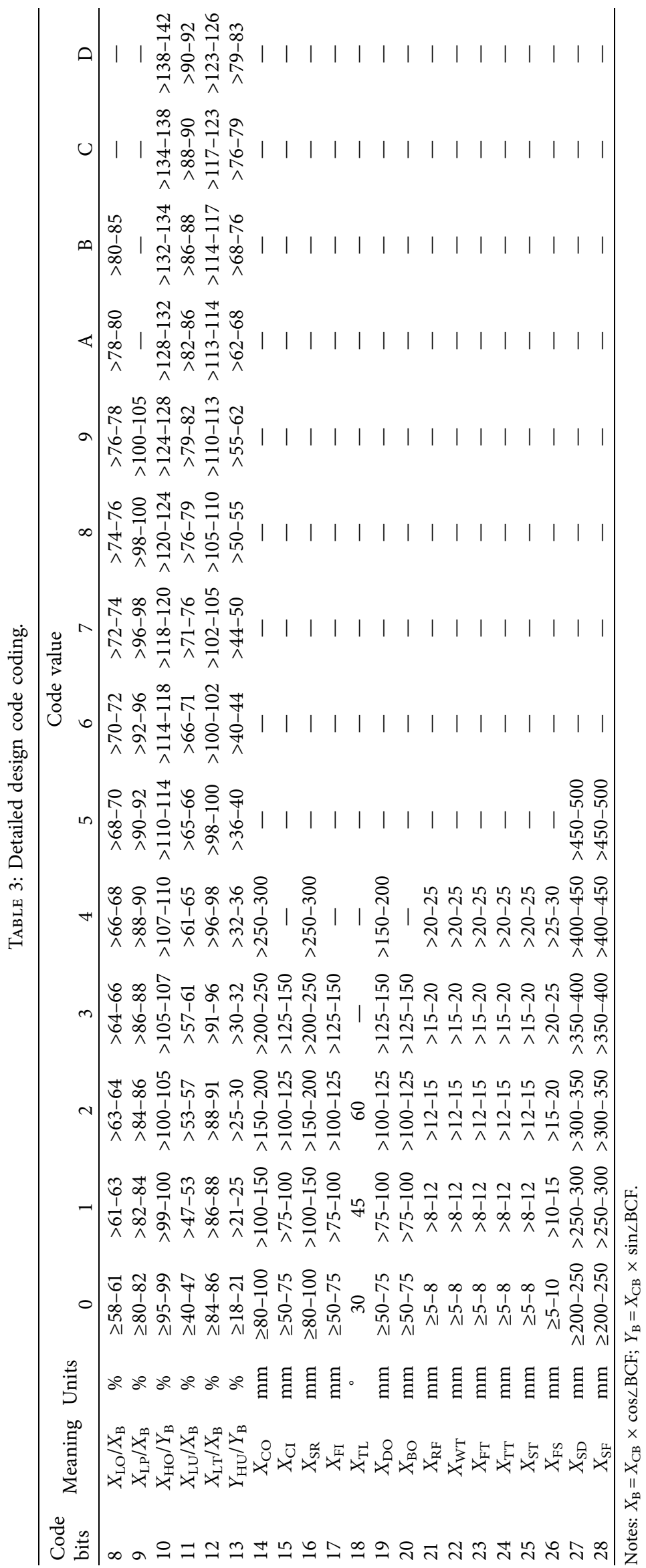




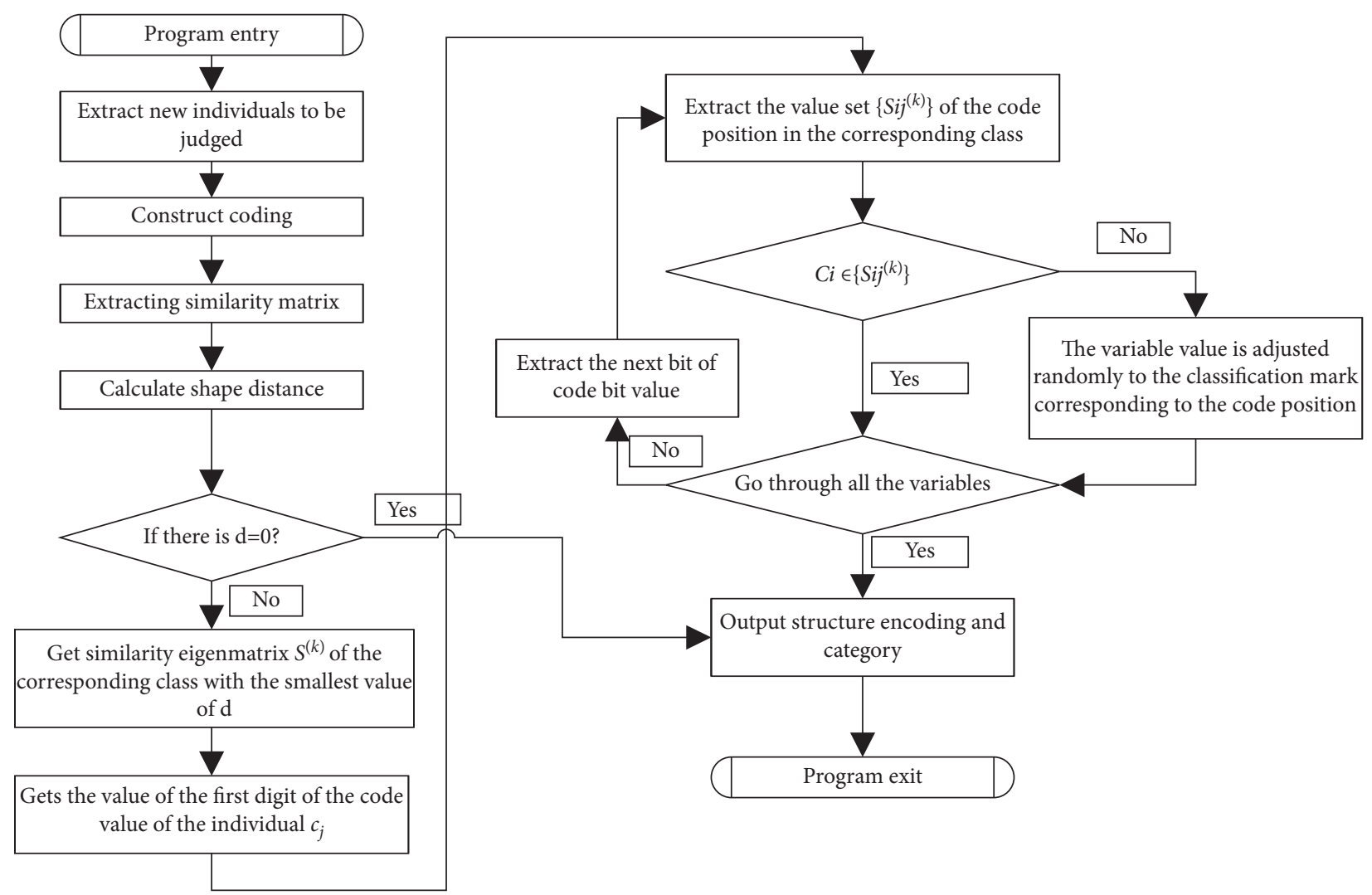

FIGURE 4: Flowchart of design knowledge processing.

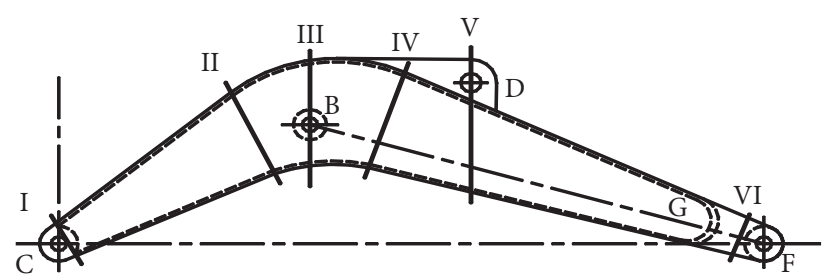

FIgURE 5: Distribution of stress characteristics of the whole gooseneck bending boom of the double-boom hydraulic cylinder.

TABLE 4: Spearman rank correlation coefficient between $X$ and $\sigma$.

\begin{tabular}{lccccccccccccc}
\hline Parameters & $X_{\mathrm{ST}}$ & $X_{\mathrm{HU}}$ & $X_{\mathrm{SD}}$ & $X_{\mathrm{RF}}$ & $X_{\mathrm{WT}}$ & $X_{\mathrm{LT}}$ & $X_{\mathrm{LO}}$ & $X_{\mathrm{BO}}$ & $X_{\mathrm{CO}}$ & $X_{\mathrm{SR}}$ & $X_{\mathrm{TT}}$ & $X_{\mathrm{HO}}$ & $X_{\mathrm{DO}}$ \\
\hline$\sigma_{\mathrm{I}}$ & -0.5 & 0.16 & -0.3 & -0.25 & -0.13 & 0.02 & 0.1 & 0.14 & -0.43 & 0.02 & -0.01 & 0.03 & -0.02 \\
$\sigma_{\mathrm{II}}$ & -0.13 & 0.74 & -0.04 & -0.11 & -0.17 & 0.12 & 0.17 & 0.1 & 0.03 & -0.13 & -0.04 & -0.06 & 0 \\
$\sigma_{\mathrm{III}}$ & -0.16 & 0.47 & 0.05 & -0.13 & 0.05 & 0.25 & 0.08 & -0.22 & 0 & 0 & -0.19 & -0.02 & -0.06 \\
$\sigma_{\mathrm{V}}$ & -0.16 & 0.33 & -0.16 & -0.08 & -0.49 & -0.04 & 0.02 & 0.16 & -0.31 & -0.01 & -0.08 & -0.05 & 0.07 \\
$\sigma_{\mathrm{IV}}$ & -0.23 & 0.27 & -0.26 & -0.54 & -0.01 & 0.11 & 0.06 & 0.14 & -0.26 & 0.03 & -0.1 & -0.05 & 0.05 \\
$\sigma_{\mathrm{VI}}$ & -0.51 & 0.25 & -0.07 & -0.05 & 0.03 & 0.2 & 0.08 & 0.13 & 0.08 & -0.33 & 0.04 & 0.13 & -0.08 \\
\hline
\end{tabular}

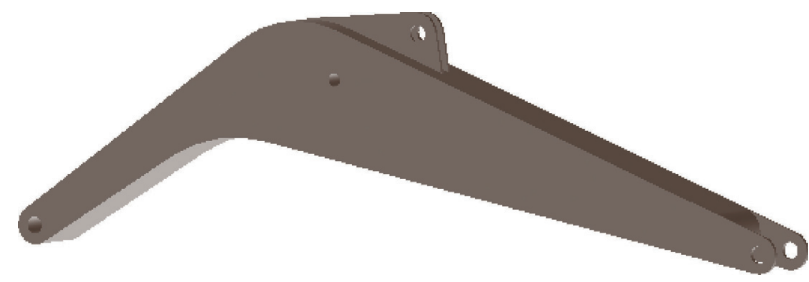

FIgURE 6: Abnormal structure boom entity diagram. 


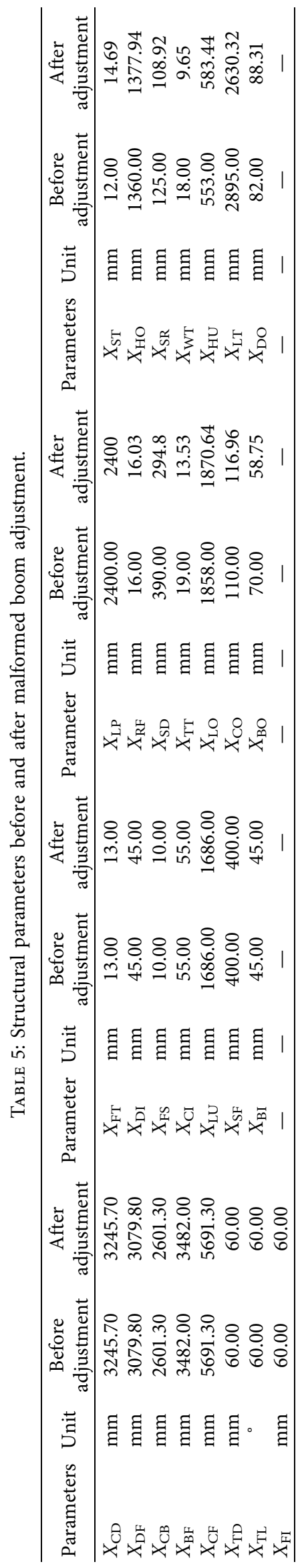




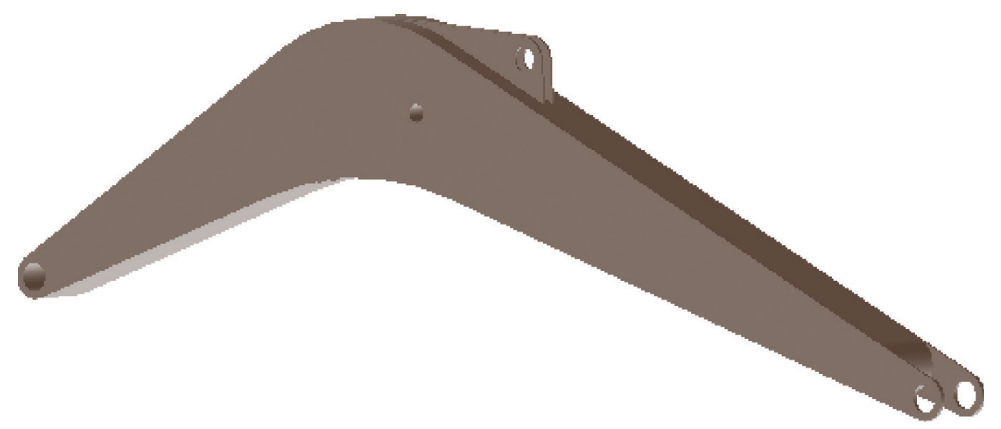

FIGURE 7: Physical diagram of the abnormal structure boom structure after rationalization.

(2) If $X_{\mathrm{ST}}$ or $X_{\mathrm{RF}}$ is increased, then $\sigma_{\mathrm{m}}$ will be decreased and $V(X)$ will be increased.

(3) If $X_{\mathrm{HO}}, X_{\mathrm{SR}}$, or $X_{\mathrm{BO}}$ is decreased, then $\sigma_{\mathrm{I}}$ and $V(X)$ will simultaneously be decreased

(4) If $X_{\mathrm{CO}}$ or $X_{\mathrm{BO}}$ is reduced, then $\sigma_{\mathrm{II}}$ and $V(X)$ will simultaneously be reduced

(5) If $X_{\mathrm{SD}}$ or $X_{\mathrm{WT}}$ is reduced, then $\sigma_{\mathrm{III}}$ and $V(X)$ will simultaneously be reduced

(6) If increasing $X_{\mathrm{LT}}$, or decreasing $X_{\mathrm{DO}}$ or decreasing $X_{\mathrm{BO}}$, then $\sigma_{\mathrm{IV}}$ and $V(X)$ will simultaneously be reduced

(7) If $X_{\mathrm{DO}}, X_{\mathrm{SR}}$, or $X_{\mathrm{BO}}$ is decreased, then $\sigma_{\mathrm{V}}$ and $V(X)$ will simultaneously be reduced

(8) If $X_{\mathrm{HO}}, X_{\mathrm{TT}}, X_{\mathrm{CO}}, X_{\mathrm{BO}}$, or $X_{\mathrm{WT}}$ reducing, then $\sigma_{\mathrm{VI}}$ and $V(X)$ will simultaneously be reduced

Based on the above conclusions, combined with the idea of intensity distribution such as stress distribution, the logical inference can be used to optimize the optimization of the boom structure.

\section{Illustrative Example}

In order to verify the feasibility and effectiveness of the malformed structure recognition technology and the malformed structure parameter adjustment technology, the boom in Figure 2 with the bucket capacity of $1 \mathrm{~m}^{3}$ is taken as the research object, and the principle of a random generation model in intelligent optimization design of the manipulator structure is simulated. The system is tested with a boom with a structural shape as an example. As shown in Figure 6, the front end of the boom structure is thick and the rear end is thin, the center of gravity is located forward, and the structure is unreasonable and unsightly, and it is a malformed structure. Therefore, it is reshaped by using the knowledge structure expression and processing mechanism. The structural parameters before and after the adjustment are shown in Table 5 . Before the adjustment, the malformed structure of boom is coded as 90011115980F7101022122122022, which cannot be correctly classified into the existing category. The system gives the judgment result of the malformed structure. After the expert knowledge adjusts the structural parameters, the structure code is 90011114980D7101022122122022, and the category is 001 . After adjustment, the physical structure of the boom structure is shown in Figure 7, and the structure is reasonable.

By comparing the parameters before and after the adjustment of the malformed structure, it can be known that, before the adjustment of the abnormal structure boom, the values of $X_{\mathrm{LU}}$ and $X_{\mathrm{LT}}$ are unreasonable with the values of $X_{\mathrm{LO}}$ and $X_{\mathrm{LP}}$, resulting in the bending of the lower cover of the boom structure. The boom structure with a thick front end and a thin back end becomes an abnormal shape. After the identification and adjustment of the expert experience knowledge, the values of $X_{\mathrm{LU}}, X_{\mathrm{LT}}$, and $X_{\mathrm{LO}}$ have changed, and the boom structure with the standard structural shape is obtained. The knowledge expression and processing mechanism of the boom structure design can effectively identify the abnormal structure generated in the creative optimization process of the boom structure, use the expert knowledge to adjust the structural parameters of the abnormal structure boom with the closest structural shape of the regular boom as a template, and achieve the goal of effectively avoiding the formation of abnormal structure booms in the optimization process.

In order to verify the effectiveness of the optimized design method of the boom structure, the structural optimization design is carried out by taking the boom shown in Figure 2 as an example. The structural parameters of the initial design, tuning shape, and optimal shape of the boom are shown in Table 6 . The initial shape structure code is 9001111A9A5C8101022122122022, and the attribution category is 001. The tuning result structure code is 9001111A9A598- 101022122122022, and the attribution category is 001 . The optimization result structure code is 9001111599585101022122122022 , and the attribution category is 004 .

Finite element analysis is performed on the initial shape, tuning results, and optimization results, and the stress distribution and volume of the boom structure are shown in Table 7. The data in the table shows that the maximum stress in the initial plan appears at the stress at position VI under the third working condition. Through structural adjustment and optimization, the maximum stress still appears at position VI under the third working condition, and the maximum stress does not exceed the original value.

Compared with the initial shape, the optimization shape optimizes the structure volume and stress distribution significantly without increasing the maximum stress of the 


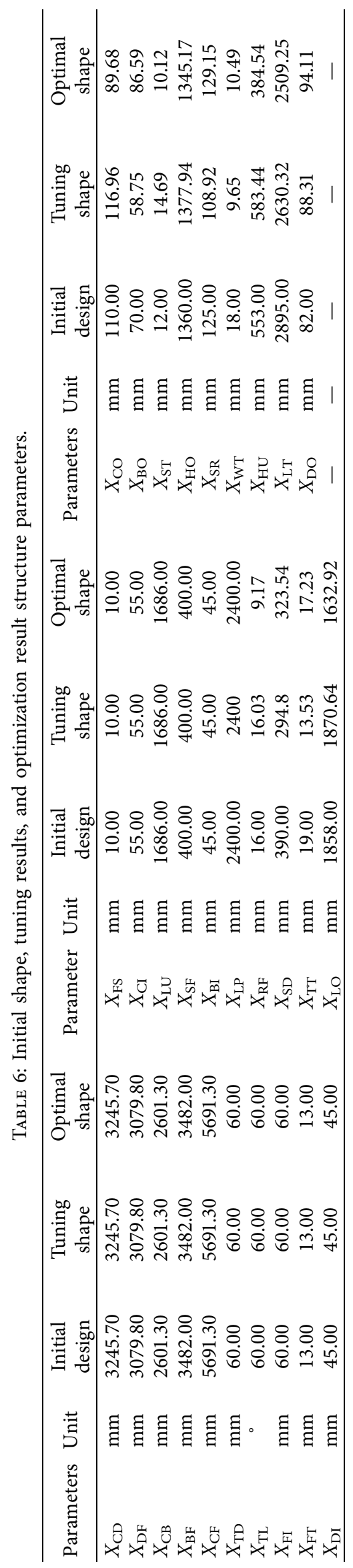


TABLe 7: Comparison of initial shape, tuning results, and optimization results.

\begin{tabular}{lcccccccccc}
\hline Shape & Condition & $\sigma_{\mathrm{I}} / \mathrm{MPa}$ & $\sigma_{\mathrm{II}} / \mathrm{MPa}$ & $\sigma_{\mathrm{III}} / \mathrm{MPa}$ & $\sigma_{\mathrm{IV}} / \mathrm{MPa}$ & $\sigma_{\mathrm{V}} / \mathrm{MPa}$ & $\sigma_{\mathrm{VI}} / \mathrm{MPa}$ & $\sigma_{\mathrm{m}} / \mathrm{MPa}$ & $V(X) / \mathrm{mm}^{3}$ & $\mathrm{Category}$ \\
\hline \multirow{3}{*}{ Initial shape } & 1 & 88.337 & 79.889 & 87.883 & 105.710 & 78.304 & 165.383 & 165.383 & \\
& 2 & 76.166 & 67.274 & 78.237 & 95.681 & 71.292 & 179.910 & 179.910 & $1.66 \times 10^{8}$ & 001 \\
& 3 & 92.538 & 93.894 & 96.410 & 119.224 & 76.112 & 242.969 & 242.969 & \\
& 4 & 90.572 & 80.348 & 87.305 & 99.560 & 76.341 & 144.800 & 144.800 & \\
Tuning result & 1 & 118.430 & 118.584 & 105.740 & 134.431 & 89.628 & 164.711 & 164.711 & \\
& 2 & 101.995 & 103.784 & 89.881 & 113.973 & 87.719 & 179.463 & 179.463 & $1.45 \times 10^{8}$ & 001 \\
& 3 & 140.735 & 142.966 & 111.769 & 141.175 & 88.438 & 241.937 & 241.937 & \\
Optimization result & 4 & 118.933 & 118.798 & 105.394 & 126.352 & 89.353 & 144.416 & 144.416 & \\
& 1 & 223.994 & 188.055 & 208.388 & 101.637 & 79.379 & 164.034 & 223.994 & \\
& 2 & 155.187 & 131.994 & 151.772 & 79.392 & 57.383 & 180.619 & 180.619 & $1.31 \times 10^{8}$ & 004 \\
& 3 & 196.277 & 183.923 & 190.165 & 98.673 & 59.494 & 241.798 & 241.798 & & \\
\hline
\end{tabular}
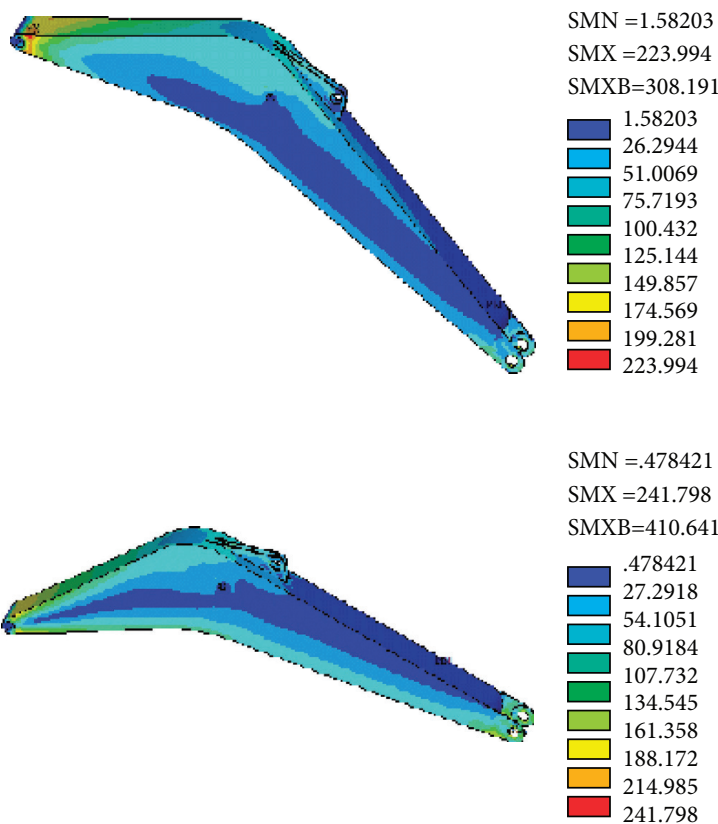
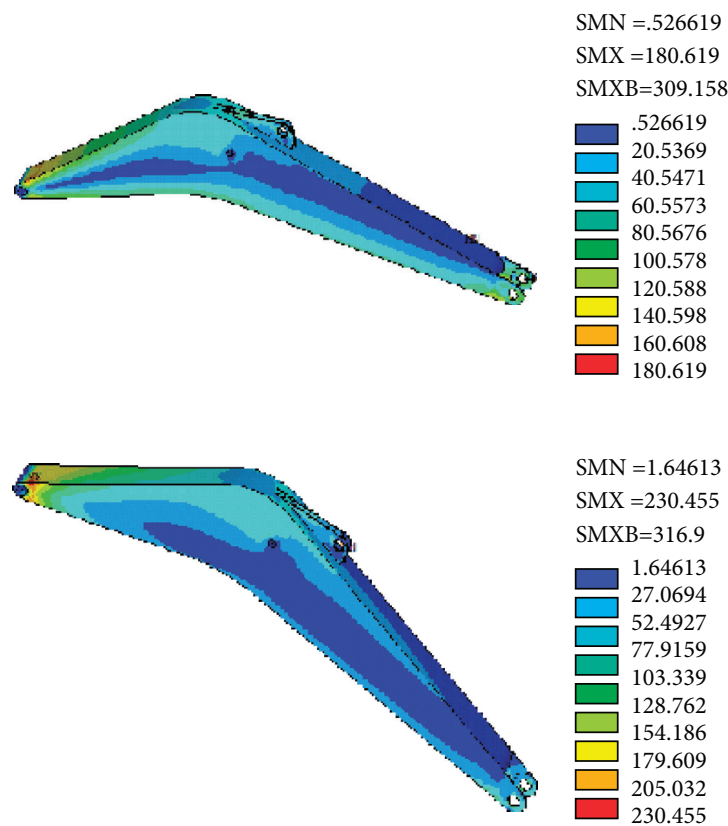

FIGURE 8: Stress distribution of optimization results under four operating conditions. (a) Working condition 1. (b) Working condition 2. (c) Working condition 3. (d) Working condition 4.

structure. In terms of structural volume, the volume of the tuning results and the optimization results is decreased to $1.45 \times 108 \mathrm{~mm}^{3}$ and $1.31 \times 108 \mathrm{~mm}^{3}$, respectively, which is decreased by $12.65 \%$ and $21.08 \%$, and the optimization results are reduced by $9.66 \%$, compared with the tuning results.

In terms of stress distribution, under the four working conditions, the maximum structural stress of the initial shape, the tuning results, and the optimization results are all present in the stress characteristic region VI under the working condition 3, but the local maximum stress and the maximum structural stress in each stress-specific region. The difference of the structure decreases and the maximum stress under the four working conditions also decreases in turn, indicating that the structural stress distribution is more uniform. Besides, the initial shape and tuning results are all 001 type booms, and the optimization result is 004 type booms, indicating that the logical reasoning tuning method is a local optimization method, and the knowledge-guided boom structure optimization can avoid falling into local optimum. The structural stress distribution diagram of the optimization results under four working conditions is shown in Figure 8.

\section{Conclusion}

In order to effectively improve the quality of structural optimization design solutions, speed up the optimization solution efficiency, and avoid structural malformations in the optimization process, this article starts with the optimization design of the boom structure, organically integrates genetic algorithms and knowledge-based systems, and performs knowledge expression and processing, structural coding, and reuse, and structural similarity evaluation was analyzed. Finally, the following conclusions are obtained: 
(1) The numerical optimization algorithm is combined with the knowledge system, and the domain optimization, design-specification knowledge, expert experience knowledge, and historical instance knowledge are used to guide numerical optimization. It had the ability to acquire, express, process, and reuse knowledge, avoid structural malformations in the optimization process and improve the efficiency and quality of optimization solutions.

(2) Based on the structure of the coding, the similarity feature matrix, and the specifications' knowledge design "shape distance" boom structure acquisition, the expression and processing mechanism can effectively identify optimize malformation boom structure generated in the process, and using the knowledge guiding-structure parameter causes deformity structure normalization.

(3) Based on Spearman rank correlation analysis, it can effectively tap the tacit knowledge in the optimization process and use logical reasoning technology to tune the boom entity in the optimization process to accelerate the optimization convergence speed.

This research combines simulation experiments with theoretical analysis and proposes a knowledge-guided optimization design method for excavator boom structures. The proposed optimization strategy is more efficient and reliable, avoids distortion during the optimization process, and speeds up the optimization of the optimization solution. Improving the quality of optimization solutions has important guiding significance. In this study, the stiffness and energy-saving performance of the boom structure were not considered in the optimization solution. Therefore, in future research, a multiobjective optimization design strategy of comprehensive strength, stiffness, and energy saving may be considered.

\section{Data Availability}

The data used to support the findings of this study are included within the article.

\section{Conflicts of Interest}

The authors declare that they have no conflicts of interest.

\section{Acknowledgments}

This work was supported by the National Foundation of Fujian Province, China (nos. 2016J01727 and 2015J01186), Fujian University of Engineering Scientific Research Launch Project (GY-Z14075), and National Fund Cultivation Project of Fujian Jiangxia University (JXZ2020003).

\section{References}

[1] L. H. Wu, J. Bai, Y. J. Zuo, G. S. Tan, Y. F. Liu, and K. T. Li, "Application of structure optimization design based on matlab genetic algorithm," Mechanical \& Electrical Engineering Technology, vol. 46, no. 10, pp. 44-47, 2017.
[2] X. Zhang, M. Huang, and C. Wang, "Development and application of radial gate optimization system based on annealing genetic algorithm," Water Power, vol. 42, no. 12, pp. 77-80, 2016.

[3] A. R. Aderiani, M. Shariatpanahi, and A. Parvizi, "Simultaneous topology and size optimization of locomotive structure using multinary genetic algorithms," Journal of Mechanical Science and Technology, vol. 31, no. 3, pp. 1283-1291, 2017.

[4] L. F. F. Miguel and L. F. Fadel Miguel, "Shape and size optimization of truss structures considering dynamic constraints through modern metaheuristic algorithms," Expert Systems with Applications, vol. 39, no. 10, pp. 9458-9467, 2012.

[5] K. Wendt, A. Cortés, and T. Margalef, "Knowledge-guided genetic algorithm for input parameter optimisation in environmental modelling," Procedia Computer Science, vol. 1, no. 1, pp. 1367-1375, 2010.

[6] C. Michaud, "New NN training algorithm and its application in optimization design," Computer Engineering \& Applications, vol. 45, no. 8, pp. 5-6, 2009.

[7] W. Zhao, F. Fan, and W. Wang, "Non-linear partial least squares response surface method for structural reliability analysis," Reliability Engineering \& System Safety, vol. 161, pp. 69-77, 2017.

[8] F. Lei and X. Han, "A study of rapid evaluation for structural behavior of multi-parameterized and largescale problem in vehicle body design," China Mechanical Engineering, vol. 6, pp. 668-671, 2010.

[9] F. Huang, X. Han, and Y. H. Huang, "Efficient method for harmonic response of structure with damping based on reduced-basis method," China Mechanical Engineering, vol. 11, pp. 1283-1288, 2010.

[10] L. L. Yang, C. Y. Chen, and D. Y. Wang, "Structural optimization of satellite-based on multi-objective collaborative optimization algorithm," Journal of Shanghai Jiaotong University, vol. 48, no. 10, pp. 1446-1450, 2014.

[11] P. Wang, S. L. Zhang, and G. Q. Wu, "Multidisciplinary design optimization of vehicle body structure based on collaborative optimization and multi-objective genetic algorithm," Journal of Mechanical Engineering, vol. 47, no. 02, pp. 102-108, 2011.

[12] H. Y. Hua and S. W. Lin, "Configurable knowledge model of structural performance characteristics for complex component," Computer Integrated Manufacturing Systems, vol. 20, no. 5, pp. 1003-1012, 2014.

[13] G. Xu, H. Ding, and Z. Feng, "Optimal design of hydraulic excavator shovel Attachment based on multiobjective evolutionary algorithm," IEEE, vol. 24, no. 2, pp. 808-819, 2019.

[14] Q. Qiu, B. Li, and P. Feng, "Optimal design of hydraulic excavator working device based on multiple surrogate models," Advances in Mechanical Engineering, vol. 8, no. 5, Article ID 168781401664794, 2016.

[15] X. Li, G. Wang, S. Miao, and X. Li, "Optimal design of a hydraulic excavator working device based on parallel particle swarm optimization," Journal of the Brazilian Society of Mechanical Sciences and Engineering, vol. 39, no. 10, pp. 3793-3805, 2017.

[16] M. J. Zhu and A. M. Ji, "Multi-objective collaborative optimization of hydraulic excavator boom," Material Engineering and Mechanical Engineering, vol. 18, pp. 228-238, 2016.

[17] Z. Zou, J. Chen, and X. Pang, "Lightweight and high-strength optimization design for a fully parametric working attachment of a hydraulic excavator based on limiting theoretical digging capability model[J]," Proceedings of the Institution of Mechanical Engineers - Part C: Journal of Mechanical Engineering Science, vol. 233, no. 14, pp. 4819-4835, 2019. 
[18] Z. H. Shen, S. Q. Yang, and P. H. Shen, "Stress distribution rapid prediction for excavator boom," Journal of Changchun University of Technology(Natural Science Edition), vol. 36, no. 4, pp. 426-432, 2015.

[19] H. Y. Hua, S. W. Lin, and Z. H. Shen, "A novel method of constraint expression and handling for complex component structural intelligent optimization," China Mechanical Engineering, vol. 25, no. 8, pp. 1085-1091, 2014. 